\title{
Research Status of Job Satisfaction of Medical Staff and its Influencing Factors
}

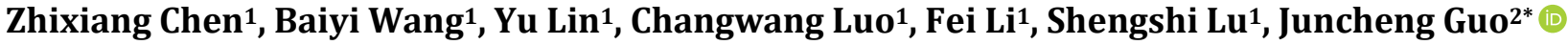 \\ ${ }^{1}$ Hainan General Hospital, Haikou, China \\ ${ }^{2}$ Central South University Xiangya School of Medical Affiliated Haikou Hospital, Haikou, China \\ Email: *g2002m@163.com
}

How to cite this paper: Chen, Z. X., Wang, B. Y., Lin, Y., Luo, C. W., Li, F., Lu, S. S., \& Guo, J. C. (2021). Research Status of Job Satisfaction of Medical Staff and its Influencing Factors. Journal of Service Science and Management, 14, 45-57.

https://doi.org/10.4236/jssm.2021.141004

Received: December 26, 2020

Accepted: February 16, 2021

Published: February 19, 2021

Copyright (c) 2021 by author(s) and Scientific Research Publishing Inc. This work is licensed under the Creative Commons Attribution International License (CC BY 4.0).

http://creativecommons.org/licenses/by/4.0/

\begin{abstract}
Human resources for health are the most important and valuable asset in an organization, and the performance of human resources is an important factor affecting the success of each organization. Hospitals play an important role in people's health and depend on human resources more than any other organization. Medical staff is the most critical factor for the development of medi$\mathrm{cal}$ and health undertakings. Therefore, how to improve the hospital's human resources, namely the satisfaction of medical staff, is a social issue in today's society. In our understanding of the present status of the medical staff, we analyze the problems existing in the hospital management, to explore the hospital at the same time of providing medical services to patients, lead to an improvement in hospital medical staff's own professional level, improve the level of hospital scientific management, scientific management and analyze the related factors, for hospital managers to provide management strategy, providing reference for improving the medical staff's work enthusiasm. On this basis, humanized management is proposed to adjust the hospital strategy, improve the medical staff's job satisfaction, further strengthen the scientific and standardized management of the hospital, improve the medical quality, and enhance the comprehensive strength of the hospital.
\end{abstract}

\section{Keywords}

Medical Personnel, Job Satisfaction, Influencing Factors, The Status Quo

\section{Introduction}

In 1935, Hoppock was the originator of formal work satisfaction studies. In his paper, Hoppock first clearly put forward the definition of job satisfaction. In his opinion, job satisfaction is a subjective response of employees to the surrounding environment of their work. Subsequently, job satisfaction has become the 
focus of competitive studies by experts at home and abroad (Hoppock, 1935). At home and abroad, a large number of studies show that high job satisfaction of medical staff is more efficient to provide medical and health services, medical staff job satisfaction and patient satisfaction, direct involvement in between (Donelan et al., 1997); the higher satisfaction of medical staff is better at bringing to the patients better medical effect (Syptak et al., 1999), helping to improve comprehensive satisfaction of patients and medical cost reduced (Katz, 1999). Compared with the developed countries, the study on the job satisfaction of medical personnel in China started late. Although in recent years, it has gradually attracted the attention of relevant scholars, the importance of its research has been increasingly recognized by peers (Chen, 2010). Therefore, how to improve the job satisfaction of medical staff is an important part of the current research of hospital managers and health decision makers. This paper discusses the relationship between the job satisfaction of medical staff and its influencing factors, as well as the influence of the influencing factors, so as to provide some data support for improving the job satisfaction of medical staff, and provide relevant theoretical basis for the formulation and improvement of medical and health policies for medical staff.

\section{The Concept of Job Satisfaction and Its Influencing Factors}

\subsection{Concept of Job Satisfaction}

Job satisfaction refers to the cognitive evaluation of an organization's members on their own work characteristics. It is the attitude and emotional experience of whether they are satisfied with all aspects of work after comparing the gap between the value they actually obtain and their expectations. Job satisfaction includes the following aspects: 1) job background satisfaction. Compensation and benefits, satisfaction with salary, bonus, subsidy, medical insurance, vacation, etc.; Working hours, reasonable on-off hours, appropriate overtime system, etc. Work space, area environment of the workplace, equipment and other resources necessary for work, etc.; 2) Work group satisfaction. Including: degree of cooperation, trust, support and guidance of superiors, mutual understanding of colleagues and comprehension ability of subordinates, and completion of tasks; Information dissemination degree, information channel smooth, accurate and efficient information transmission; Understanding of the work unit, understanding and recognition of its history, values, strategies, etc.; Organizational participation, whether opinions and suggestions are valued, participation in decision-making, etc. The change of job satisfaction, to some extent, reflects some problems of hospital management decision. At the same time, it is also an effective method to develop democratic decision-making and management in hospitals. Therefore, the investigation on the job satisfaction of medical staff not only conforms to the international and domestic "people-oriented" environment, but also conforms to the in-depth development of the "hospital Management year" activity. The earliest concept of job satisfaction originates from the Hawthorne 
Experiment, co-led by Mayo, Roethisberger \& Whitehead, a Western electrical company in Chicago, from 1927 to 1932. The study points out that workers' emotions affect their work behavior, and workers' social and psychological factors are the main factors that determine their job satisfaction and productivity (Huang, 2003). The formal study of job satisfaction started from Hoppock (1935), the famous book Job Satisfaction, which first put forward the concept of job satisfaction. He believed that job satisfaction refers to the satisfaction of workers to environmental factors in both psychological and physiological aspects, that is, the subjective response of workers to the work situation (Hoppock, 1935). Since then, job satisfaction has become a topic that many scholars have been competing to discuss. When studying job satisfaction, different theoretical frameworks will be adopted according to different research objects, so the definition of job satisfaction will be different. Porter \& Lawler III (1968) believed that the degree of job satisfaction was determined by the gap between a person's actual reward and the reward he thought he deserved. The gap was small, but the degree of satisfaction was large. On the contrary, a large gap means a small degree of satisfaction (Chen et al., 2003). Vroom (1973) believed that job satisfaction generally refers to the feeling or emotional reaction of the role of the job itself in the organization (Green et al., 2000). Cavanagh (1989) believes that job satisfaction is the degree to which an individual likes his job as shown. Robbins (1997) believed that job satisfaction is the general attitude of workers towards their work, and a high degree of job satisfaction indicates that workers have a positive attitude towards their work. There are also many domestic scholars who define job satisfaction: Xu Fuming and Shen Jiliang (2001) put forward the overall job satisfaction, which refers to a worker's attitude towards the work itself, the working environment and other factors. Lu et al. (2001) believed that job satisfaction is a reflection of employees' attitude towards their work or work experience evaluation, which is different from satisfaction in life and personal career development.

\subsection{Influencing Factors of Job Satisfaction of Medical Staff}

\subsubsection{Degree}

In China, higher education does not mean higher job satisfaction of medical staff. After entering the medical institutions, the income and professional title of college graduates are not high, but some medical personnel with low education background are much higher in income and professional title than those with high education background because they entered the institutions earlier. On the other hand, highly educated medical workers have a huge gap between their expected income and actual income, which to some extent leads to the phenomenon that the more educated they are, the lower their job satisfaction will be.

\subsubsection{Income}

The higher the salary, the higher the job satisfaction of the medical staff. Medical workers with higher incomes face greater risks and work pressure, especially 
those with annual income between 48,000 and 60,000 yuan, which leads to lower job satisfaction to a certain extent.

\subsubsection{Types of Medical Institutions}

In China, the satisfaction rate of medical workers in community health is the highest, followed by that of medical workers in township hospitals, and the satisfaction rate of medical workers in hospitals is the lowest. From the point of view of medical staff most want to improve, in addition to income as the first aspect, hospital medical staff most eager to improve the doctor-patient relationship. At the same time, in addition to the income conditions of the medical staff in the community health service center, the most urgent thing is to improve the working ability of individuals.

\subsubsection{Professional Category}

Medical staff of nursing profession in China had the highest satisfaction degree, followed by medical staff of public health, and medical staff of clinical medical profession had the lowest satisfaction degree. On the one hand, it is because medical staff in clinical medical profession face higher work pressure than medical staff in nursing and public health profession. On the other hand, nursing and public health professionals have a better doctor-patient relationship than clinical medical professionals.

\subsubsection{Department}

The satisfaction rate of medical staff in Chinese medicine is the highest, followed by preventive health department, and the satisfaction rate of medical staff in surgery department is the lowest. This is mainly because the average weekly working hours of medical staff in traditional Chinese medicine and preventive health services are much lower than those in surgery.

\subsubsection{Working Intensity}

The longer the working hours per week, the lower the job satisfaction of medical staff; The higher the number of night shifts per month, the lower the job satisfaction of medical staff; The greater the work stress, the lower the job satisfaction of medical staff. Considering that medical workers serve people's precious lives with strong operability and high requirements on knowledge and experience, the working pressure they face is much higher than that of the general population. Because working pressure is too big, when you have little medical staff can get the support of the resources, only a lot into their own resources to meet the requirements of their own, such as increasing their emotional labor, will own psychological feelings to control in such aspects as facial expressions, language and behavior (Cheng \& Mao, 2014), and this kind of long time of emotional labor is easy to make the medical staff to produce physical and psychological diseases, cause misdiagnosis and medical staff at the time of diagnosis and misdiagnosis, endanger the patient's life and health. In this case, the satisfaction of medical workers is bound to be affected to different degrees (Liang et al., 2014). 


\subsubsection{Degree of Respect between Patients and Society}

The premise of a harmonious doctor-patient relationship is mutual respect between doctors and patients (Shen et al., 2013). Patients' respect for medical staff is conducive to improving the patience of medical staff, satisfying their psychology of being valued, confirming their ability, and thus stimulating the work enthusiasm of medical staff. Due to the excessive exaggeration of social media, many people in the society have a bias in understanding and evaluation of medical personnel, which leads to the decline of social respect for doctors, which is not conducive to the construction of a good medical practice environment. Good social respect is helpful to improve the self-esteem and pride of medical staff and improve their job satisfaction, for example, doctors take kickbacks on drugs, consumables and laboratory tests.

\subsubsection{Patient Trust}

The higher the patient's trust in the medical staff, the higher the satisfaction of the medical staff. Patients' high trust in doctors is the recognition and recognition of medical staff's medical technical level, which can greatly encourage the confidence of medical staff. Meanwhile, the higher the patient's trust in medical staff, the more conducive to the communication and cooperation between doctors and patients, so as to improve the job satisfaction of medical staff.

\subsubsection{Patient Satisfaction}

Medical staff's perception of their work has a significant impact on the quality of medical staff's work (Amiresmaili \& Moosazadeh, 2013). Whether the patient is satisfied or not, the medical staff can directly feel. In addition, high patient satisfaction is also a great affirmation and encouragement for medical staff's medical technology and services, which can improve their job satisfaction to a certain extent.

\subsubsection{Doctor-Patient Relationship}

Good doctor-patient relationship, let the medical staff do not need to pay too much in front of the patients with emotional labor, which can make the medical staff will be more focused on clinical skills, and improve medical service quality and work efficiency, promote the effective implementation of the medical intervention, to ensure the effect of medical intervention, effectively promote the doctor-patient communication on both sides; However, poor doctor-patient relationship may not only hinder the treatment of patients, but also endanger the personal safety and social stability of medical staff in the long run.

Medical personnel are closely related to human health. In the current situation, research the medical staff's job satisfaction status, and its influencing factors is of great significance, deeply and put forward effective improvement measures and policy recommendations, eventually improve the medical staff job satisfaction, this to improve the quality of medical service environment and the society as a whole is of great significance, benefit is the entire social group. 


\section{Countermeasures to Improve Medical Staff Satisfaction}

Studies on job satisfaction of medical staff at home and abroad show that medical staff with high job satisfaction can provide better health services than those with low job satisfaction, and there is a direct correlation between job satisfaction of medical staff and patient satisfaction. A highly satisfied medical staff is likely to produce good outcomes for patients, which will contribute to improving patients' overall satisfaction and reducing costs, thus positioning the hospital in terms of competitiveness, medical market share and profit margins. However, the negative impact of the decreased job satisfaction of medical staff is mainly reflected in the following aspects: it leads to the increase of medical staff turnover rate and mental disorders, affects the continuity of providing health care services for patients, tension between medical loyalty, increased medical disputes, and rising cost of health service system, etc. (Zhao, 2016). There were statistically significant differences between different groups of medical workers in terms of social status, work remuneration, working environment, management system, professional development and security. In order to improve the job satisfaction of medical staff, the following countermeasures should be taken according to their specific problems:

\subsection{Construction of Medical Staff Satisfaction Evaluation System}

At present, there are many ways to measure job satisfaction, and the most widely used means to investigate job satisfaction are the single holistic assessment method and the comprehensive scoring method composed of multiple job elements. In the general field of job satisfaction, there are many classic assessment tools based on different theories and concepts, such as satisfaction scale and job description index scale, job diagnosis scale, job satisfaction scale, Peter needs Satisfaction questionnaire, etc. Development and research is suitable for the characteristics of the medical personnel professional job satisfaction scale, standardization and normalization, through the satisfaction assessment, and for deviation in the management of the hospital prevention effect, become an early prediction of hospital management instrument, can diagnosis the present situation of the hospital management, monitoring the effectiveness of the hospital performance management can also be measured in advance know the flow of the medical staff turnover intention, prevent the loss of excellent medical personnel, to ensure the sustainable development of health (Yang, 2014). The establishment of medical staff satisfaction evaluation system and understanding of the job satisfaction of medical staff can clarify the problems existing in hospital management, so as to develop corresponding measures to improve the satisfaction of medical staff. The satisfaction survey results are also the most important "thermometer" to diagnose the current situation of hospital management and provide scientific reference and basis for hospital management decision-making. Through understanding the factors that affect the job satisfaction of clinical medical staff, it is of great significance and significance to improve the job satisfaction of clin- 
ical medical staff, care about their physical and mental health, and reflect the concept of "people-oriented" in modern management to promote the construction of a harmonious society in China.

\subsection{Establish a Reasonable Remuneration System to Improve the Remuneration of Medical Staff}

Relevant studies in China show that salary level is related to job satisfaction, and satisfaction with salary increase also increases. Hospital managers should consider the difference of salary standards of different professions, and consider the income of doctors from the overall perspective of society (Xue et al., 2014). A reasonable reward system should be established, which includes material reward and non-material reward. Material remuneration includes direct remuneration (such as salary, bonus, etc.) and indirect remuneration (such as welfare, paid leave, etc.); Non-material remuneration includes professional rewards (such as practice, further study, etc.) and social rewards (such as promotion, promotion, evaluation of excellence, etc.). Should be reasonable income gap, formulate reasonable appraisal, rewards and punishments mechanism ( $\mathrm{Hu}, 2013)$, abandon the pot and equalitarianism, implements the dynamic management, various personnel post once change, allowance changed accordingly, true " $F$ do to post pay. Heavy performance and contribution distribution mechanism is established. According to efficiency first, fairness considered, the principle of paying equal attention to economic and social benefits, the hospital internal distribution to make significant achievements and contributions to highlight and key positions of staff tilt, with full reflect the value and status of the talent (Liu et al., 2014). Doctor reasonable income increase involves many deep-seated problems, It needs to be gradually solved by the state in the reform, such as the annual salary system, raising the price of diagnosis and treatment, and setting the salary of posts according to the technical difficulty, so that their income is proportional to the risk and pressure they bear.

\subsection{Provide Professional Development Opportunities and Improve Job Satisfaction}

There are three ways to improve the working ability of medical personnel in China: learning through work, learning through communication and professional training. Hospital managers should create a working environment for staff to learn from and discuss with each other. Measures to improve doctor satisfaction are not limited to the return on a doctor's salary. Opportunities for career development are equally important. Hospitals should formulate professional development plans, vigorously support discipline development, and gradually implement key discipline development plans at the national, provincial and hospital levels. Asked to further strengthen domestic and international academic exchanges, for doctors to provide more learning opportunities and the chance of the professional communication between peers will also improve the doctor's 
degree of satisfaction, especially the need to provide them with some practical measures and the system and be implemented, such as various departments to declare national and regional (provincial) provide fast and convenient service to carry on the work of education mode, but also to improve national and regional (provincial) continuing medical education project director reward standard. To further improve the management system of continuing medical education, improve the website distance education model, encourage experts to actively participate in the development of continuing medical education projects, in addition, some experts can be hired for targeted training. Training is a kind of emotional investment, which can induce the gratitude of medical staff, and express the care, attention and help for medical staff, as well as the high hopes and expectations for the future development of medical staff.

\subsection{Improve the Competitiveness of Human Resources and Mobilize the Work Enthusiasm of Medical Staff}

At present, the serious shortage of nurses in China has become a common phenomenon in all levels of hospitals. Behind the serious shortage of nurses is the decrease of the overall medical service quality of the hospital, which makes the medical service quality decline seriously and causes the increase of medical hidden trouble. According to a clinical nurse shortage problem, hospital managers should strengthen and emphasize the clinical nursing work, scientific arrangement of the nurse resources, to recognize proverbs \{to nurse clinical service main body, is to respect the nurse, tap potential, effectively play a role of nurses, improve the nurses working environment, improve the treatment, so as to improve medical service quality and job satisfaction. The core competitiveness of modern hospitals is human resources, and the competitiveness of human resources is to improve the social and economic benefits of hospitals by improving the value of human capital (Tong \& Luo, 2015). Hospital managers should not only make full use of hospital human resources, but also pay attention to the development and protection of human resources. The hospital should regard medical personnel as the main object of humanistic management and the most important resource of the hospital, respect individual value, fully develop human resources and improve the competitiveness of human resources. The hospital should establish a set of perfect, feasible, and easy to operate human resources incentive mechanism, establish a multi-level, multi-structure, multi-sequence medical talent system, and build a platform for all kinds of talents to display their aspirations, so as to discover talents, train talents, introduce talents, respect talents, reuse talents and retain talents. In order to retain talents, hospitals must create an environment of caring, loving, using, cultivating, understanding and trusting people. Hospital managers should set up the strategic concept of human resources, establish the development plan of human resources; To create a highly efficient, standardized, preferential and dynamic policy environment; Adhere to the correct orientation of choose and employ persons, to create open, fair, equal- 
ity, competition and merit of candidate mechanisms of choose and employ persons, the technical level is high, strong leadership, the personnel selection of noble medical ethics to important positions: establish talent selection, cultivation, management, use, evaluation method (Sun, 2013), such as to ensure talent management standardized, scientific, healthy and orderly direction. To establish the law on evaluation and appointment of professional and technical positions related to talents and measures for personnel exchange; create a business learning and communication environment that is conducive to the growth and development of talents. Organize staff to learn the advanced technology and management mode of hospitals at home and abroad, broaden their vision and make innovations and development. Care about people's physical and mental health, constantly improve the material and cultural life of staff, strengthen the construction of hospital hardware facilities, at the same time care about the health of medical staff, regular physical examination, and for the staff to handle hospitalization insurance, relieve their worries, to create a healthy and comfortable working and living environment; And the establishment of a scientific and reasonable salary distribution system and incentive mechanism, etc. (Gu et al., 2016), to fully mobilize the work enthusiasm, initiative and creativity of medical staff, so as to improve their job satisfaction.

\subsection{Implement Humanistic Management Scientifically and Establish Effective Management System}

Based on the comprehensive description and analysis of the current situation of job satisfaction of medical staff at home and abroad, it can be found that compared with people in other professions, medical staff are under great pressure and have great risks in work, and their feelings and attitudes towards work are somewhat different from those in other professions (Ding \& Sun, 2014). Hospital managers in hospital policy and implementation management, must consider the hospital medical staff is the main body of this factor, in all aspects of hospital management reflects the features of humanistic management, not only to consider the differences between different groups, should also focus on clinical front line staff tilt, especially in the paid work, work, work environment, management system, hospital culture, such as treatment teaching material and spiritual "staff first, can the patient first" the idea, and closely around the idea to implement hospital management (Xie, 2017). At the same time, on the basis of fully understanding the needs of medical personnel, we should meet their reasonable requirements and "respect people". We should fully mobilize their enthusiasm and "liberate people". Improve their quality and skills, create an innovative environment for their "development of people". The hospital should establish human-oriented management mechanism, that is, implement emotional communication management, medical staff participate in management, medical staff self-management, public management and hospital culture management, so that the majority of workers can personally feel higher satisfaction, more participa- 
tion, less mental pressure.

\subsection{Establish a Harmonious Working Environment to Improve the Safety of Medical Staff}

We should correctly guide the news public opinion, publicize the main theme, actively publicize the medical personnel to heal the wounded and rescue the dying, implement the spirit of social justice, humanism and selfless dedication, and create good public opinion. To keep the message, channel unobstructed, to ensure the timely, accurate and efficient information dissemination; 1) To provide a comfortable working environment and optimize the working environment for medical personnel; 2) To be equipped with necessary medical equipment and other resources; We should protect the legitimate rights and interests and personal safety of medical personnel by legal means, and take effective measures to curb the phenomenon of "medical trouble", so as to improve the satisfaction of medical personnel in terms of social status, working environment and sense of security. Due to the nature of clinical work, there are certain risks, and clinical medical staff may be harmed by the personal and personal dignity of some patients or their family members, and they have to face medical disputes, which makes the job security of medical staff decline. It is suggested that hospital administrators should strengthen psychological counseling for clinical medical staff and purchase insurance for surgeons to protect their physical and mental health. In addition, the hospital's quality control work is extended to the link of receiving patients' complaints, timely discovering medical quality problems, solving problems in a targeted way, and preventing medical disputes (He, 2016).

\subsection{Create a Harmonious Hospital Cultural Atmosphere and Improve the Core Competitiveness of the Hospital}

In the face of the fierce competition in the medical market, the current hospital managers are more and more deeply aware that cultural management has become the mainstream trend of the development of hospital management, which is a powerful means to enhance the core competitiveness of hospitals and ensure the sustainable development of hospitals. According to Maslow's five-level theory of needs, what hospital workers need more is the embodiment of social recognition and self-worth (Sun et al., 2014). This requires hospital managers to reflect the people-oriented management concept in the management work. The emphasis of cultural management should be placed on people, especially on people's values, to stimulate the sense of responsibility and enthusiasm of employees, and to emphasize the integration of people and work, individuals and hospitals. Hospital culture is not only an idea, a management philosophy, but also a kind of power. It has a strong appeal, centripetal force, guiding force, cohesion and binding force for hospital staff, and can enhance staff's trust, pride and sense of honor in the hospital. Through the construction of hospital culture, cultivate the common values of all the staff, use various incentive means, fully mobilize and give play to the enthusiasm and creativity of people, guide all the 
staff to achieve the hospital's development goals, rely on the joint efforts of all the staff, promote the hospital's continuous development. The hospital is a high-tech, highly knowledgeable place, as well as a department with high quality and high service. For this reason, medical staff prefer to work in a working environment that is people-oriented, respects human nature and fosters advanced hospital culture. They hope to have a flexible and relaxed working space and a interpersonal atmosphere of kindness, solidarity and cooperation. Because such an atmosphere can bring people a happy emotional state, high enthusiasm for work, more business achievements, the motivation to work consciously. Therefore, managers should attach importance to creating such a cultural atmosphere, which is also an aspect of the core competitiveness of hospitals (Cavanagh, 1989).

\section{Conclusion}

In a word, the analysis of medical staff's job satisfaction enables hospital administrators to find problems existing in management and take corresponding measures. In short, after analyzing the job satisfaction of medical staff, hospital administrators can find the problems existing in management and take corresponding measures accordingly. We should care about medical staff, improve the level of professional knowledge of medical staff, to meet the reasonable living requirements of medical staff, hospital managers in the realization of providing high-quality medical level for patients at the same time. We should care about the medical staff, show the care and concern for the medical staff, arouse the enthusiasm of the medical staff, stimulate the professional dedication of the medical staff, establish the self-esteem of the medical staff, make the medical staff take the initiative to provide patients with satisfactory medical services, and make contributions to the development of the hospital. We will continue to implement the policy of deepening reform of the medical and health care systems. Multiple measures are taken to protect the legitimate rights and interests of medical personnel and improve their satisfaction; implement the three-medical linkage reform in the fields of medical treatment, medical insurance and medicine to improve patient satisfaction; continue to promote the construction of close medical alliance; to make up for the shortcomings in the implementation of graded diagnosis and treatment, improve accounting policies according to local conditions, and give appropriate policy preference to grassroots medical and health institutions. Therefore, hospital managers should pay attention to improving the job satisfaction of medical staff, so as to improve the quality of medical services and hospital management level.

\section{Foundation Project}

Hainan Health Commission Science and Technology Project (No. 20A200228).

\section{Conflicts of Interest}

The authors declare no conflicts of interest regarding the publication of this paper. 


\section{References}

Amiresmaili, M., \& Moosazadeh, M. (2013). Determining Job Satisfaction of Nurses Working in Hospitals of Iran: A Systematic Review and Meta-Analysis. Iranian Journal of Nursing and Midwifery Research, 18, 343-348.

Cavanagh, S. J. (1989). Nursing Turnover: Literature Review and Methodological Critique. Journal of Advanced Nursing, 14, 587-596. https://doi.org/10.1111/j.1365-2648.1989.tb01595.x

Chen, G. H., Fang, H., \& Liu, C. Y. (2003). Organizational Behavior. Beijing: Tsinghua University Press.

Chen, L. (2010). Research on Job Satisfaction of Urban Community Doctors and Its Influencing Factors. Shenyang: China Medical University.

Cheng, C. L., \& Mao, H. R. (2014). How to Rebuild Healthy and Harmonious Doctor-Patient Relationship. China Health and Nutrition, 24, 1788-1789. (In Chinese)

Ding, L., \& Sun, H. (2014). Trial of Trait Emotional Intelligence Questionnaire in Medical Staff. Chinese Clinical Psychology, 19, 470-472. (In Chinese)

Donelan, K., Blendon, I. L., Lundberg, G. D. et al. (1997). The New Medical Marketplace: Physicians' Views. Health Affairs, 16, 139-148. https://doi.org/10.1377/hlthaff.16.5.139

Green, J. et al. (2000). Job Satisfaction of Community College Chairpersons. The Faculty of the Virginia Polytechnic Institute and State University. Doctor of Philosophy, Virginia: The Faculty of the Virginia Polytechnic Institute and State University.

Gu, S. T., Huang, Q. M., Chen, Z. Q. (2016). A Study on the Relationship between Job Satisfaction and Turnover Intention of Commercial Bed Doctors in Grade III Hospitals. Chinese Journal of Hospital Management, 22, 586-589.

He, J. F. (2016). A Study on the Job Satisfaction of Medical Staff. Journal of Shanxi Medical University, 36, 709-711.

Hoppock, R. (1935). Job Satisfaction. New York: Harper \& Row.

$\mathrm{Hu}, \mathrm{W}$. (2013). Discussion on Influencing Factors of Clinicians' Job Satisfaction. Chinese Hospital Statistics, 20, 7-9.

Huang, X. Y. (2003). A Study on Job Satisfaction and Its Influencing Factors. Tianjin: Tianjin Normal University.

Katz, A. (1999). Better Outcome Means More Job Satisfaction: Pilot Project in Winnipeg and Halifax to Enhance Physician-Patient Communication. Canadian Family Physician, 45, 218-220.

Liang, Z. J., Wang, R., Wang, S. et al. (2014). Influence of Doctor-Patient Interaction on Job Satisfaction and Turnover Intention of Medical Staff. Medicine and Society, 27, 8-9.

Liu, L. Y., Jiang, C. Y., Dong, Z. J. et al. (2014). Investigation on Factors Affecting the Enthusiasm of Medical Staff and Countermeasures under the New Situation. Chinese Medical Ethics, 27, 422-424.

Lu, J., Shi, K., \& Yang, J. F. (2001). The Evaluation Structure and Method of Job Satisfaction. Human Resources Development in China, No. 1, 15-17. (In Chinese)

Porter, L. W., \& Lawler III, E. E (1968). Managerial Attitudes and Performance. Homewood, IL: R.D. Irwin.

https://www.worldcat.org/title/managerial-attitudes-and-performance/oclc/243614

Robbins, S. (1997). Organizational Behavior (7th ed.). Beijing: Renmin University of China Press.

Shen, Y. M., Niu, S. M., Zhang, Y. et al. (2013). Analysis of Reasons for Lack of Doc- 
tor-Patient Trust and Reconstruction of Trust. Chinese Hospital Management, 33, 79-80.

Sun, H., Ding, L., \& Liu, F. R. (2014). A Study on the Correlation between Trait Emotional Intelligence and Job Satisfaction in Medical Staff. Chinese Hospital Management, 32, 58-60.

Sun, J. H., Shi, Y., \& Li, Y. Q. (2013). Measurement and Evaluation of Medical Staff's Job Satisfaction. Medicine and Society, 19, 57-59.

Syptak, J. M., Marsland, D. W. et al. (1999). Job Satisfaction Putting Theory into Practice. Family Practice Management, 6, 26-31.

Tong, L. L., \& Luo, H. (2015). Study and Analysis of Medical Staff's Job Satisfaction. Chinese Hospital Management, 23, 7-8.

Vroom, V., \& Yetton, P. (1973). Leadership and Decision-Making. Pittsburgh, PA: University of Pittsburgh Press. https://doi.org/10.2307/j.ctt6wrc8r

Xie, J. Q. (2017). Discussion on Physician Performance Evaluation and Incentive Mechanism. Chinese Journal of Health and Digestion, 8, 389-396.

Xu, F. M., Shen, J. L. (2001). Study on Job Satisfaction of Primary and Secondary School Teachers and Its Countermeasures. Educational Science Research, No. 9, 23-26.

Xue, R., Yuan, S. Z., \& Zhang, B. (2014). Job Satisfaction Survey and Demand Analysis of Medical Staff. Chinese Hospital Management Miscellany, 21, 756-758.

Yang, J. F. (2014). Evaluation Structure and Method of Job Satisfaction. China Human Resources Development, No. 1, 15-17.

Zhao, S. M. (2016). New Progress in Human Resource Management Research (pp. 267-287). Nanjing: Nanjing University Press. 